\title{
Emulator development and USB implementation on SDKP16
}

\begin{abstract}
System development kit (SDK) is an important tool for software testing of developed processors and it has wide applications in integrated circuit (IC) production as well as in education sectors. It can be used as a learning kit for engineering or computer science students to learn programming languages. In this project, a SDK developed by MIMOS named SDKP16 is investigated and improved to ease its usage. The improvements implemented in this project were upgrading its serial port to USB port for communication purpose and development of Visual Basic program as its communication terminal on the host instead of using Hyper Terminal. The challenges in this project include the integration of the USB port into the existing SDK board and test of the developed program of which to observe the USB protocols. The project has increased compatibility and usability features of the SDKP16.
\end{abstract}

Keyword: Software testing; Utility program; Microcprocessor 